\title{
Correlation of Creatine Kinase Levels with Clinical Features and Survival in Amyotrophic Lateral Sclerosis
}

\author{
Hongfei Tai ${ }^{1}$, Liying Cui ${ }^{1,2 *}$, Yuzhou Guan', Mingsheng Liu' ${ }^{1}$, Xiaoguang Li ${ }^{1}$, Dongchao \\ Shen ${ }^{1}$, Dawei Li ${ }^{1}$, Bo Cui', Jia Fang ${ }^{1}$, Qingyun Ding ${ }^{1}$, Kang Zhang ${ }^{1}$ and Shuangwu Liu ${ }^{1}$ \\ ${ }^{1}$ Department of Neurology, Peking Union Medical College Hospital, Chinese Academy of Medical Sciences, Peking Union \\ Medical College, Beijing, China, ${ }^{2}$ Neuroscience Center, Chinese Academy of Medical Sciences, Beijing, China
}

OPEN ACCESS

Edited by: Ghazala Hayat,

Saint Louis University, United States

Reviewed by: James Gilchrist,

Southern Illinois University

School of Medicine,

United States

Howard Sander,

New York University School of Medicine, United States

*Correspondence:

Liying Cui

pumchcuily@yahoo.com

Specialty section:

This article was submitted to Neuromuscular Diseases, a section of the journa Frontiers in Neurology

Received: 02 May 2017 Accepted: 20 June 2017 Published: 03 July 2017

Citation:

Tai H, Cui L, Guan Y, Liu M, Li X, Shen D, Li D, Cui B, Fang J, Ding Q. Zhang K and Liu S (2017) Correlation of Creatine Kinase Levels with Clinical

Features and Survival in Amyotrophic Lateral Sclerosis. Front. Neurol. 8:322. doi: 10.3389/fneur.2017.00322
Objective: To evaluate serum creatine kinase (CK) levels of amyotrophic lateral sclerosis (ALS) patients and to explore the relationship between CK levels and the clinical characteristics and survival prognosis of ALS patients.

Methods: We analyzed the CK levels of 185 ALS patients who underwent long-term follow-up. The relationship between CK levels and clinical features including sex, age, disease duration, site of onset, body mass index (BMI), serum creatinine (Cr), and spontaneous electromyographic activity was analyzed by univariate analysis and multiple linear regression. Kaplan-Meier and Cox proportional hazards models were used to explore whether CK levels were independently correlated with survival prognosis of ALS.

Results: Baseline serum CK was raised in $43 \%$ of participants. The median CK level was $160 \mathrm{U} / \mathrm{L}$ (range: 20-2,574 U/L), and 99\% of patients had a baseline serum CK level less than 1,000 U/L. CK levels were significantly higher in male patients than in female patients [204 (169) versus 117 (111) U/L, $p<0.001$ ] and in patients with limb onset ALS than with bulbar onset ALS $(p<0.001)$. CK levels were also correlated with serum $\mathrm{Cr}$ $(p=0.011)$ and the spontaneous potential score of electromyography (EMG) $(p=0.037)$ but not correlated with age $(p=0.883)$, disease duration $(p=0.116)$, or BMI $(p=0.481)$. Log CK was independently correlated with survival of ALS patients $(H R=0.457,95 \%$ confidence interval $0.221-0.947, p=0.035$ ) after adjusting for age, sex, site of onset, serum $\mathrm{Cr}$, and $\mathrm{BMI}$.

Conclusion: Serum CK levels of ALS patients were correlated with sex, site of onsite, serum $\mathrm{Cr}$, and spontaneous activity in EMG. Serum CK could be an independent prognostic factor for survival of ALS patients.

Keywords: amyotrophic lateral sclerosis, creatine kinase, clinical characteristics, survival, prognosis

\section{INTRODUCTION}

Amyotrophic lateral sclerosis (ALS) is a fatal neurodegenerative disorder involving primarily motor neurons in the cerebral cortex, brainstem, and spinal cord that is characterized by progressive generalized muscle weakness, muscular atrophy, and upper motor neuron signs. The etiology remains unclear, and ALS is generally diagnosed based on clinical manifestations combined with an electrophysiological exam. 
Several clinical laboratory tests could be abnormal in typical ALS, such as muscle enzyme creatine kinase (CK). Serum CK has been considered to be associated with muscle damage and has been used to differentiate myopathic lesions from neurogenic lesions. Since the 1970s, several studies have reported that CK could be mildly to moderately elevated in ALS patients (1-10), although mechanism of CK elevation in ALS is still not well understood. Several studies showed significantly higher CK levels in male ALS patients than in female patients $(3,4,10)$ and in limb onset ALS compared to bulbar onset patients $(3-5,9$, $10)$, but some studies did not find any difference between sexes $(5,6)$ and sites of onset $(6)$. Furthermore, some prior studies found no relationship between CK levels and survival of ALS patients $(3,4,11)$, although one recent study showed that low CK levels were associated with longer survival (9), and another found a positive correlation between CK levels and survival (10). Given these controversial findings, this study was conducted to describe serum CK levels in Chinese ALS patients and to explore the factors influencing CK concentration and whether it is a predictor of survival of ALS patients.

\section{MATERIALS AND METHODS}

This was a retrospective observational cohort study that included 185 ALS patients registered at Peking Union Medical College Hospital from January 2013 to December 2015. Patients fulfilled the revised El Escorial criteria (12) for clinically definite, probable or laboratory supported probable ALS were recruited. Patients with a concurrent acute infection, liver disease, or kidney disease were excluded.

The demographic features and clinical data of patients were collected, including age, sex, site of onset, disease duration, height, weight, and body mass index (BMI). Patients were followed up periodically by phone calls or face-to-face interviews, and survival status was last updated in December 2016. Survival time was calculated as the time from the date of enrollment to death or tracheotomy.

The venous blood samples of all patients were drawn after overnight fasting and rest. CK concentration was measured by a direct enzymatic method according to the International Federation of Clinical Chemists. The normal range of serum CK concentrations in our center is defined as $24-195 \mathrm{U} / \mathrm{L}$ in men and $24-170 \mathrm{U} / \mathrm{L}$ in women. Serum creatinine $(\mathrm{Cr})$ was also tested in the same sample.

Electromyography (EMG) data at the time of diagnosis were also reviewed in 147 patients at our center using unique criteria. The amount of spontaneous potentials (SPs) in EMG was semiquantitatively scaled from 1 to 10 points according to the locations and intensity of the fibrillation potential, the positive sharp wave and fasciculation potentials in the tested muscles, including the sternocleidomastoid, biceps brachia, triceps brachia, extensor digitorum communis, abductor digiti minimi, abductor pollicis brevis, quadriceps femoral, tibialis anterior, gastrocnemius, and paraspinal muscles. Then, the mean SP score of all muscles was calculated for each person.

This study was approved by the Ethics Committee of Clinical Research of Peking Union Medical College Hospital (Beijing, China), and all patients signed informed consents.

\section{Statistical Analysis}

Creatine kinase concentrations were presented as the median (quartiles) due to the non-normal distribution. The Wilcoxon/ Mann-Whitney $U$-test or the Kruskal-Wallis test was used to detect the correlation between serum CK concentrations and clinical features provided as categorical variables, such as gender and site of onset. Spearman correlation analysis was used to assess quantitative variables, including age, BMI, disease duration, serum $\mathrm{Cr}$, and SP score. In addition, the $\mathrm{CK}$ data were log transformed to generate a normal distribution. A multivariate linear regression model was used to detect any independent effect of these clinical factors on the log CK values. The Kaplan-Meier method was used to generate survival curves for patients with different CK levels (normal or raised) and compared using the log-rank test. Univariable and multivariable analyses were performed with the Cox proportional hazards $(\mathrm{PH})$ model to examine the effect of baseline $\log \mathrm{CK}$ on the survival outcome after adjusting for confounding factors. A $p$-value of $<0.05$ was regarded as statistically significant. Statistical analyses were performed using the SPSS 21.0 software.

\section{RESULTS}

A total of 185 patients (107 males, 78 females) were included in this study, and the mean (SD) age was 53 (12) years (range 20-77 years). The patients were predominantly limb onset ALS with a mean duration of 16 months from disease onset to study entry. The baseline clinical features of the participants were shown in Table S1 in Supplementary Material.

\section{Serum CK Levels in Patients with ALS}

The median CK level for the entire group was $160 \mathrm{U} / \mathrm{L}$ (range 20-2,574 U/L), and the mean CK value was $218 \mathrm{U} / \mathrm{L}$. Forty-three percent of patients had an abnormal CK concentration higher than the upper limit of the normal range. Ninety-nine percent of the participants had a CK level less than 1,000 U/L with only two patients with a CK level above 1,000 U/L.

\section{Relationship between CK Levels and Clinical Characteristics of ALS}

Table 1 presents the serum CK levels of patients in different subgroups. CK levels were significantly higher in male patients than

TABLE 1 | Serum creatine kinase (CK) levels of patients in different gender and site of onset.

\begin{tabular}{llrcc}
\hline & Subgroups & $\boldsymbol{n}$ & $\begin{array}{c}\text { Median CK (quartile, } \\
\text { range), U/L }\end{array}$ & $\boldsymbol{p}$-Value \\
\hline Overall & & 185 & $160(160,20-2,574)$ & \\
By gender & Male & 107 & $204(169,20-2,574)$ & $<0.001$ \\
& Female & 78 & $117(111,25-858)$ & \\
By site of onset & Upper limb & 64 & $171(152,30-2,574)$ & $<0.001$ \\
& Lower limb & 80 & $197(146,32-1,153)$ & \\
& Bulbar & 33 & $92(43,25-376)$ &
\end{tabular}


in female patients $(p<0.001)$. The percentage of male patients with raised CK levels (57/107) was also higher than that in female patients $(22 / 78)$ (Pearson's chi-square, $p=0.001)$. The $\mathrm{CK}$ value in upper or lower limb onset ALS was higher than in bulbar onset ALS $(p<0.001)$, but there was no significant difference between the upper and lower limb onset groups $(p=0.144)$. Univariate analysis also revealed a significant correlation between CK levels and serum $\mathrm{Cr}$ concentration $(r=0.208, p=0.005)$, EMG SP score $(r=0.200, p=0.015)$, and duration of illness $(r=0.168$, $p=0.022)$ but no correlation with patient age $(p=0.883)$ or BMI $(p=0.671)$.

Multivariate linear regression model showed that log CK was independently correlated with sex $(p=0.001)$, site of onset $(p<0.001)$, serum Cr $(p=0.011)$, and EMG SP score $(p=0.037)$,

TABLE 2 | Factors significantly correlated with log CK in ALS patients by multivariate linear regression analysis.

\begin{tabular}{lccccr}
\hline Variables & $\begin{array}{c}\text { Estimated } \\
\text { parameter }(\boldsymbol{\beta})\end{array}$ & SE & 95\% Cl & $\boldsymbol{T}$ & $\boldsymbol{p}$-Value \\
\hline Sex & 0.177 & 0.054 & $0.070-0.284$ & 3.260 & 0.001 \\
Site of onset & 0.254 & 0.063 & $0.129-0.378$ & 4.033 & $<0.001$ \\
Serum Cr & 0.005 & 0.002 & $0.001-0.009$ & 2.580 & 0.011 \\
SP score & 0.025 & 0.012 & $0.002-0.049$ & 2.110 & 0.037 \\
\hline
\end{tabular}

$\mathrm{Cl}$, confidence interval; $\mathrm{Cr}$, creatinine; $\mathrm{ALS}$, amyotrophic lateral sclerosis; $\mathrm{CK}$, creatine kinase; SP, spontaneous potential. but not correlated with duration of illness $(p=0.116)$ or BMI $(p=0.481)$ (Table 2).

\section{Association between Serum CK and Survival in ALS Patients}

Amyotrophic lateral sclerosis-related deaths occurred in 74 patients through December 2016. Kaplan-Meier survival curves were drawn after dividing all subjects into two groups based on normal CK and raised CK levels (Figure 1). The log-rank test indicated that patients with raised CK had significant longer survival than patients with normal CK levels $(p=0.030)$.

The Cox $\mathrm{PH}$ model was then utilized to examine the association between $\log \mathrm{CK}$ and survival prognosis in ALS with or without adjusting for other covariates (Table S2 in Supplementary Material). Log CK was confirmed to be significantly associated with overall survival (Table 3 ). There was a $54.3 \%$ reduction in risk of death with each $1 \mathrm{U}$ increase in $\log \mathrm{CK}$ [adjusted $\mathrm{HR}=0.457,95 \%$ confidence interval $(\mathrm{CI})$ $0.221-0.947, p=0.035$.

\section{DISCUSSION}

Creatine kinase is an enzyme expressed by various tissues and cell types, which catalyzes the reversible conversion of creatine and

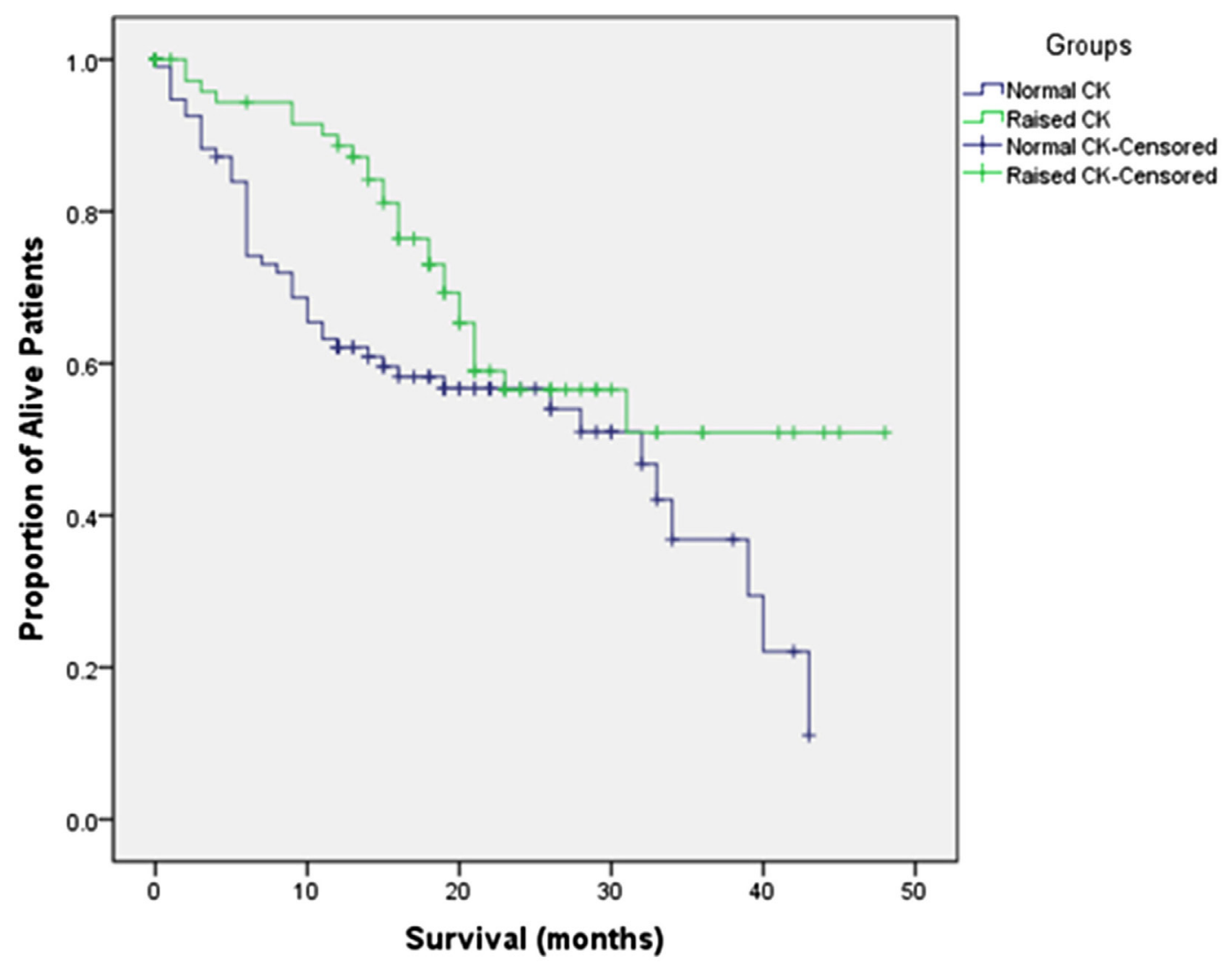

FIGURE 1 | Kaplan-Meier survival curves for amyotrophic lateral sclerosis patients with normal creatine kinase (CK) versus raised CK levels. 
TABLE 3 | Cox proportional hazards survival analysis results.

\begin{tabular}{llcr}
\hline & & HR (95\% Cl) & p-Value \\
\hline Model I & Log CK (unadjusted) & $0.347(0.172-0.704)$ & 0.003 \\
Model II & Log CK (adjusted) & $0.457(0.221-0.947)$ & 0.035
\end{tabular}

$H R$, hazard ratio; CK, creatine kinase.

${ }^{a}$ Adjusted for age, sex, site of onset, disease duration, serum creatinine, and body mass index.

utilizes adenosine triphosphate (ATP) to create phosphocreatine and adenosine diphosphate. In tissues that consume ATP rapidly, especially skeletal muscle, CK is an important enzyme. Clinically, serum CK is assayed as a marker of CK-rich tissue damage such as in muscular disease, myocardial infarction, and acute kidney injury.

Raised serum CK concentrations are also found in ALS patients. In the present study, $43 \%$ of ALS patients had a CK concentration above the upper limit of the normal range. This is consistent with previous studies that reported $23-70 \%$ of ALS patients could have raised CK levels (2, 3, 5-10). In total, 90\% of participants less than $400 \mathrm{U} / \mathrm{L} \mathrm{CK}$, and $99 \%$ had less than $1,000 \mathrm{U} / \mathrm{L} \mathrm{CK}$ corresponding to all but two cases. Unlike in muscle diseases, ALS patients usually have mildly or moderately raised $\mathrm{CK}$ levels.

The mechanism of CK elevation in ALS has not been clearly elaborated. To date, the main hypotheses are as follows: (1) muscle energy metabolism disturbance may cause increased endogenous ATP activity in mitochondria, resulting in upregulated CK expression to provide an energy substrate (10). (2) Significantly elevated CK is associated with increased muscle cell membrane permeability due to denervated muscle and historically myopathic changes in ALS patients (13).

Several studies have shown higher serum CK levels in male ALS patients than in female patients $(3,4,10)$, which is consistent with our findings. However, Gibson's study (9) showed that the difference in CK between genders was no longer statistically significant after adjusting for bioelectric impedance spectroscopy measured fat free mass, indicating that the difference in CK values by gender could be explained by differences in muscle mass. Rafiq's study (10) also revealed a linear correlation between serum CK and lean body mass estimated by the Boer formula. We also found a positive correlation between serum CK and Cr levels $(p=0.011)$, which is considered as a surrogate of muscle mass (14). This evidence suggests that, to a certain extent, higher CK levels might be associated with greater muscle mass. However, no studies, including the present study, directly measured the muscle mass of ALS patients using a precise instrument, which should be evaluated in future studies.

In addition, a dramatic loss of CK activity has been detected in transgenic ALS animal studies $(15,16)$. In G93A transgenic mice, CK activity in homogenates from spinal cords decreased to $49 \%$ and in mitochondrial fractions to $67 \%$ compared to CK activity in wild-type control mice. CK enzyme was crucial for energy metabolism and maintaining ATP levels, it might be reasonable for the compensatory upregulation of $\mathrm{CK}$ in this condition.
Our study confirmed that CK levels in limb onset ALS were significantly higher than those in bulbar onset ALS. It has been hypothesized that increased CK in ALS might reflect the extent of muscle involvement. As SPs in EMG recordings are a reliable sign of progressive muscle denervation, only one study has examined the association between CK levels and the number of muscles with fasciculation or fibrillation potentials in EMG recordings, although no relationship was found (6). For the first time, we developed an SP score taking into account the extent and amount of SPs in each muscle, and all muscles examined in every region and revealed that the $\mathrm{CK}$ value was associated with SP scores. This may support the muscle damage hypothesis to explain the raised CK mechanism. We supposed that upregulation of CK expression and muscle denervation may both have independent effects on CK levels according to the multivariable linear analysis.

There was a direct correlation between CK level and disease duration ( $r=0.168, p=0.022)$, but it became not significant in multivariate regression analysis $(p=0.116)$. Then we added the variables into the model step by step, the significance of "duration" turned to be unremarkable when "site of onset" was in. That is to say, the difference in CK level by duration could be explained by differences in site of onset, and no independent correlation was found between CK level and duration of illness as in prior studies $(5,10)$.

The influence of serum CK on survival prognosis of ALS patients has been controversial. Two prior studies did not find a significant relation between CK levels and survival of ALS patients by the Kaplan-Meier log-rank test or direct correlation analysis $(3,4)$. These studies might be limited due to the small sample ( $n=30$ and $n=73$ ). Two additional studies conducted recently demonstrated conflicting results. One study showed that ALS patients with high baseline CK (>200 U/L) had a significantly higher hazard of death than patients with low baseline CK ( $\leq 200 \mathrm{U} / \mathrm{L})$ after adjusting for age, gender, race, BIS fat free mass, location of onset, and study site (HR $=3.57,95 \%$ CI $1.84-7.27$, $p<0.05$ ) (9). This is contrast to our results. Of note, the 68 participants in Gibson et al.s study were recruited at five study centers with different reported upper limits of normal CK from each laboratory site (ranging from 145 to $320 \mathrm{U} / \mathrm{L}$ ), but in the survival analysis, CK was considered as dichotomous variable by the same cutoff value of $200 \mathrm{U} / \mathrm{L}$. We supposed the differences among laboratory tests and selection of the cutoff value may have influenced the study results; furthermore, the baseline disease duration was not adjusted for, which may also give rise to bias. The other study found that higher $\mathrm{CK}_{\log }$ was significantly associated with better survival, even after adjusting for other prognostic variants $(\mathrm{HR}=0.74,95 \%$ CI $0.59-0.93, p=0.013$ ) (10). This report is consistent with our findings, indicating that serum CK levels are positively associated with ALS survival and could be an independent marker in ALS studies and clinical drug trials. As CK was log transmitted in the Cox $\mathrm{PH}$ analysis, we should cautiously interpret the result, and the effect of the original CK value on survival should not be exaggerated. On the other hand, the positive effect on survival also supported the protective mechanism hypothesis for raised CK, such as upregulated expression. 
Due to the retrospective design of the study and missing data, we could not examine the relationships between CK values and the slope of ALS-FRS-R scores, forced vital capacity, and cramps. The extent and severity of cramps could also be related to CK elevation (9) and may be associated with the site of onset. Because of the short follow-up time for patients censored, the sample for the survival analysis was relatively small, and long-term observations are still needed. In addition, the association between CK and muscle mass should be confirmed with accurately tested muscle mass data. Although we suggest that some clinical features are related to $\mathrm{CK}$ and raised $\mathrm{CK}$ levels might be an overall protective factor, molecular-level studies that directly demonstrate the mechanism of CK elevation are warranted.

\section{ETHICS STATEMENT}

This study was approved by the Ethics Committee of Clinical Research of Peking Union Medical College Hospital (Beijing, China), and all patients signed informed consents.

\section{REFERENCES}

1. Panitch HS, Franklin GM. Elevation of serum creatine phosphokinase in amyotrophic lateral sclerosis. Neurology (1972) 22(9):964-6. doi:10.1212/ WNL.22.9.964

2. Harrington TM, Cohen MD, Bartleson JD, Ginsburg WW. Elevation of creatine kinase in amyotrophic lateral sclerosis. Potential confusion with polymyositis. Arthritis Rheum (1983) 26(2):201-5. doi:10.1002/art.1780260212

3. Sinaki M, Mulder DW. Amyotrophic lateral sclerosis: relationship between serum creatine kinase level and patient survival. Arch Phys Med Rehabil (1986) 67(3):169-71. doi:10.1016/0003-9993(86)90064-X

4. Felice KJ, North WA. Creatine kinase values in amyotrophic lateral sclerosis. J Neurol Sci (1998) 160(Suppl 1):S30-2. doi:10.1016/S0022-510X(98)00195-6

5. Ilzecka J, Stelmasiak Z. Creatine kinase activity in amyotrophic lateral sclerosis patients. Neurol Sci (2003) 24(4):286-7. doi:10.1007/s10072-003-0161-8

6. Lima AF, Evangelista T, de Carvalho M. Increased creatine kinase and spontaneous activity on electromyography, in amyotrophic lateral sclerosis. Electromyogr Clin Neurophysiol (2003) 43(3):189-92.

7. Sussmuth SD, Tumani H, Ecker D, Ludolph AC. Amyotrophic lateral sclerosis: disease stage related changes of tau protein and S100 beta in cerebrospinal fluid and creatine kinase in serum. Neurosci Lett (2003) 353(1):57-60. doi:10.1016/j.neulet.2003.09.018

8. Chahin N, Sorenson EJ. Serum creatine kinase levels in spinobulbar muscular atrophy and amyotrophic lateral sclerosis. Muscle Nerve (2009) 40(1):126-9. doi:10.1002/mus.21310

9. Gibson SB, Kasarskis EJ, Hu N, Pulst SM, Mendiondo MS, Matthews DE, et al. Relationship of creatine kinase to body composition, disease state, and longevity in ALS. Amyotroph Lateral Scler Frontotemporal Degener (2015) 16(7-8):473-7. doi:10.3109/21678421.2015.1062516

10. Rafiq MK, Lee E, Bradburn M, McDermott CJ, Shaw PJ. Creatine kinase enzyme level correlates positively with serum creatinine and lean body mass, and is a prognostic factor for survival in amyotrophic lateral sclerosis. Eur J Neurol (2016) 23(6):1071-8. doi:10.1111/ene.12995

\section{AUTHOR CONTRIBUTIONS}

HT: conception of the work, data acquisition, statistical analysis, and writing of the first draft. LC: conception and organization of the work, manuscript review, and critique. YG, ML, and XL: clinical and electrophysiological evaluation of patients. DS, DL, BC, JF, QD, KZ, and SL: data acquisition, long-time follow-up of patients, and statistical analysis.

\section{FUNDING}

This work was supported by CAMS Innovation Fund for Medical Sciences (CIFMS, 2016-12M-1-004).

\section{SUPPLEMENTARY MATERIAL}

The Supplementary Material for this article can be found online at http://journal.frontiersin.org/article/10.3389/fneur.2017.00322/ full\#supplementary-material.

11. Chio A, Calvo A, Bovio G, Canosa A, Bertuzzo D, Galmozzi F, et al. Amyotrophic lateral sclerosis outcome measures and the role of albumin and creatinine: a population-based study. JAMA Neurol (2014) 71(9):1134-42. doi:10.1001/jamaneurol.2014.1129

12. Brooks BR, Miller RG, Swash M, Munsat TL. El Escorial revisited: revised criteria for the diagnosis of amyotrophic lateral sclerosis. Amyotroph Lateral Scler Other Motor Neuron Disord (2000) 1(5):293-9. doi:10.1080/ 146608200300079536

13. Achari AN, Anderson MS. Myopathic changes in amyotrophic lateral sclerosis. Pathologic analysis of muscle biopsy changes in 111 cases. Neurology (1974) 24(5):477-81. doi:10.1212/WNL.24.5.477

14. Thongprayoon C, Cheungpasitporn W, Kashani K. Serum creatinine level, a surrogate of muscle mass, predicts mortality in critically ill patients. J Thorac Dis (2016) 8(5):E305-11. doi:10.21037/jtd.2016.03.62

15. Wendt S, Dedeoglu A, Speer O, Wallimann T, Beal MF, Andreassen OA. Reduced creatine kinase activity in transgenic amyotrophic lateral sclerosis mice. Free Radic Biol Med (2002) 32(9):920-6. doi:10.1016/S0891-5849 (02)00784-0

16. Pierce A, Mirzaei H, Muller F, De Waal E, Taylor AB, Leonard S, et al. GAPDH is conformationally and functionally altered in association with oxidative stress in mouse models of amyotrophic lateral sclerosis. J Mol Biol (2008) 382(5):1195-210. doi:10.1016/j.jmb.2008.07.088

Conflict of Interest Statement: The authors declare that the research was conducted in the absence of any commercial or financial relationships that could be construed as a potential conflict of interest.

Copyright (c) 2017 Tai, Cui, Guan, Liu, Li, Shen, Li, Cui, Fang, Ding, Zhang and Liu. This is an open-access article distributed under the terms of the Creative Commons Attribution License (CC BY). The use, distribution or reproduction in other forums is permitted, provided the original author(s) or licensor are credited and that the original publication in this journal is cited, in accordance with accepted academic practice. No use, distribution or reproduction is permitted which does not comply with these terms. 